\title{
Computation and Analysis of Power System Voltage Oscillations from Interarea Modes
}

\author{
Luigi Vanfretti, Student Member, IEEE, and Joe H. Chow, Fellow, IEEE
}

\begin{abstract}
Most studies of power system interarea mode oscillations focus on the analysis of slow coherent groups of synchronous machine angle and speed variables. It has not been clearly defined how the interarea modes of oscillation propagate through the power network. The purpose of this paper is to bridge this gap by studying the oscillations present in the bus voltage and frequency variables. Such knowledge would be useful for using phasor measurement data on high-voltage transmission buses. Earlier a technique has been proposed to develop an interarea model of a power transfer path using synchronized phasor data. Here the technique to obtain voltage and frequency oscillation characteristics is generalized to multi-machine power systems with multiple interarea modes.
\end{abstract}

Index Terms-Interarea oscillation, voltage phasors, modal analysis, linear analysis techniques, voltage oscillations.

\section{INTRODUCTION}

$\mathbf{M}$ ost studies of power system interarea mode oscillations focus on the analysis of slow coherent groups of synchronous machine angle and speed variables [1], [2], [3], [4], [5]. It has not been clearly defined how the interarea modes of oscillation propagate through the power network. The purpose of this paper is to bridge this gap by studying the oscillations present in the bus voltage and frequency variables. Such knowledge would be useful for using phasor measurement data on high-voltage transmission buses. Earlier a technique [6] has been proposed to develop an interarea model of a power transfer path using synchronized phasor data. Here the technique to obtain voltage and frequency oscillation characteristics is generalized to multi-machine power systems with multiple interarea modes.

Interarea modes of active power oscillations are determined by groups of synchronous machines interacting via the power network. As a result, machine angles and speeds tend to have clear modal contents that can be seen from simulation and actual measurements. These interarea modes will manifest in the network variables, that is, voltages and currents, as well. These network variables have received less attentions because they are also subject to reactive power control equipment, such as generator excitation systems and static var systems. The voltage response near a large load center can be influenced by the load composition. However, at transmission buses with no nearby generators and loads, interarea modes can be clearly observable from the bus voltage variables [7]. Furthermore, any jumps in the voltage magnitude can be filtered so only the oscillatory modes are kept. An analysis of the voltage variables is important because phasor measurements can be

L. Vanfretti and J. H. Chow are with the Department of Electrical, Computer, and Systems Engineering, Rensselaer Polytechnic Institute, Troy, NY 12180, USA. (e-mail: vanfrl@rpi.edu, chowj@rpi.edu).

978-1-4244-4241-6/09/\$25.00 @2009 IEEE more readily available from transmission substations than generating substations.

This paper generalizes the bus voltage and frequency analysis in [6] and [8] to multi-machine systems. The work in [6] starts with an electromechanical model of a two-machine system and uses a perturbation analysis to examine the voltage and frequency mode shapes along the impedance between the two machines. For a multi-machine power system, we start from the linearized model with bus voltages and frequencies as output variables. The columns of the output matrix in modal form then become the voltage and frequency oscillations of the interarea modes. This idea is illustrated using both a two-machine and a four-machine system, each with a single interarea mode. A more complex example is a six-machine system with two interarea modes. Such an analysis offers new understanding on how interarea modes propagate through a power network.

The remainder of the paper is organized as follows. Section II reviews the results in [6] and [8] for a two-machine system. Section III provides a modal analysis for bus voltages and frequencies in a multi-machine system. Illustrations of the proposed method are shown in Sections IV, V and VI.

\section{Voltage And Frequency OSCILlations IN TWO-MACHINE SYSTEMS}

The technique developed in [6] uses two basic physical observations of power systems discussed in [8]. By exploiting these observations, the dynamic parameters of a two-machine power system, such as the one shown in Fig. 1, are estimated.

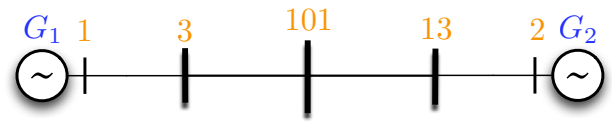

Figure 1. Two-Machine Power System

The first observation is that disturbances at dominant high voltage transfer paths can cause multiple cycles of oscillations on the bus voltages at a single interarea mode. This has been seen from simulated disturbance responses [8], and more recently confirmed from phasor measurement data originating in high-voltage transmission buses [7]. The bus voltage oscillations are used within a reactance extrapolation algorithm to estimate the internal reactances in the two-machine system. This algorithm provides a Jacobian function, when this function is plotted as the reactace of the transfer path is varied it generates a curve, namely the Jacobian fit, as shown in Fig. 2. The Jacobian function, being a derived representation of the bus magnitude voltage oscillations, provides a measure of 


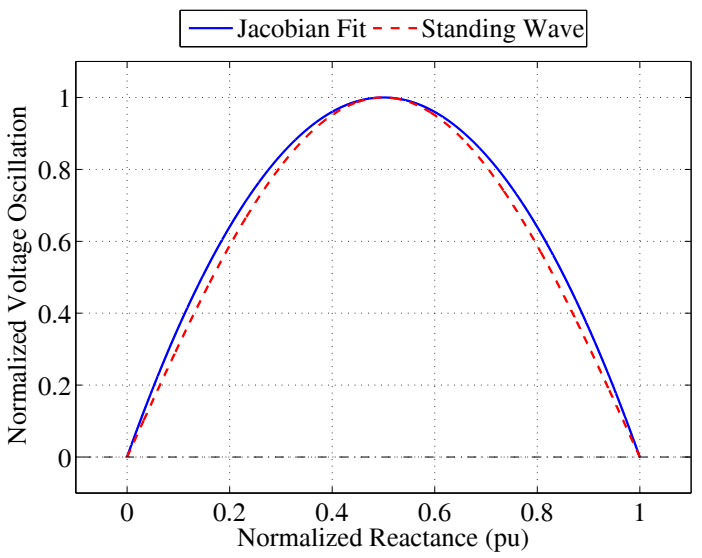

Figure 2. Normalized Voltage Oscillations

the variation of the voltage oscillations across a transfer path and estimates the voltage fixed points in the network. We will show in the following sections how, by numerically computing the voltage magnitude oscillations, similar results are obtained for more complex power systems.

The second observation to recognize that the angular momentum in a two-machine power system is related to the machine inertias and the machine speed, and that by using linearization, the total equivalent inertia constant of the power system can be estimated from the interarea frequency of oscillation. Furthermore the individual machine inertias can be obtained from an estimate of the machine speeds derived from the bus frequencies [6], [8]. Note that the bus frequencies are derived variables from the bus voltage angles. The inertia extrapolation algorithm uses this observation to obtain the machine inertias by using the derived bus frequencies from the bus voltage angle phasor measurements. The variation of these frequencies across the transfer path is typified by the curve shown in Fig. 3. In the next sections we will show that by using a new analytical method, frequency oscillation curves (similar to the one in Fig. 3) can be obtained for complex multimachine networks.

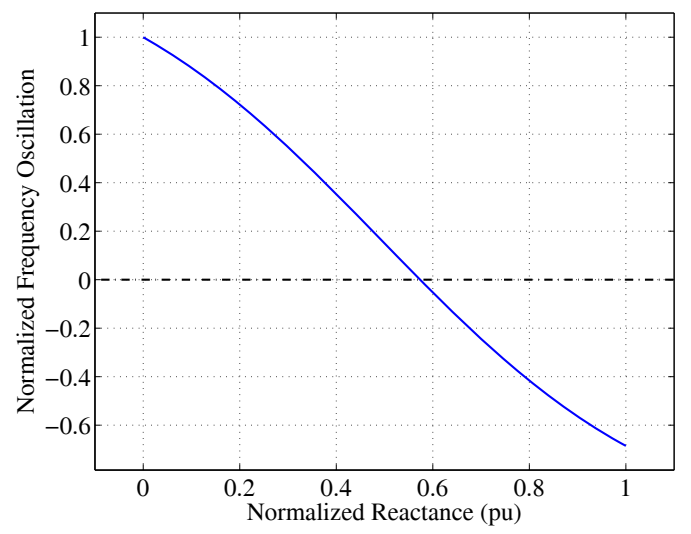

Figure 3. Normalized Frequency Oscillations

\section{An Analytical Method for Computing Oscillations in Multi-Machine Power Systems}

\section{A. Power System Linearized Model}

The linearized model of an power system is given by

$$
\begin{aligned}
& \dot{\boldsymbol{x}}_{\mathrm{P}}=\boldsymbol{A}_{\mathrm{P}} \boldsymbol{x}_{\mathrm{P}}+\boldsymbol{B}_{\mathrm{P}} \boldsymbol{u}_{\mathrm{P}} \\
& \boldsymbol{y}_{\mathrm{P}}=\boldsymbol{C}_{\mathrm{P}} \boldsymbol{x}_{\mathrm{P}}+\boldsymbol{D}_{\mathrm{P}} \boldsymbol{u}_{\mathrm{P}}
\end{aligned}
$$

where $\boldsymbol{A}_{\mathrm{P}}$ is the system matrix, $\boldsymbol{B}_{\mathrm{P}}$ the input matrix, $\boldsymbol{C}_{\mathrm{P}}$ the output matrix, $\boldsymbol{D}_{\mathrm{P}}$ the feedforward matrix, $\boldsymbol{x}_{\mathrm{P}}$ the state vector, $\boldsymbol{u}_{\mathrm{P}}$ the control vector, and $\boldsymbol{y}_{\mathrm{P}}$ the output vector.

If the model of the power system is the minimal electromechanical model for an $\mathrm{N}$-machine network, then the state vector will be given by

$$
\boldsymbol{x}_{\mathrm{P}}=\left[\begin{array}{lllllll}
\delta_{1} & \ldots & \delta_{N} & \omega_{1} & \ldots & \omega_{N}
\end{array}\right]^{T}
$$

where $\delta_{i}$ and $\omega_{i}$ are the $i$-th machine internal angle and speed respectively.

The output matrix $C_{\mathrm{P}}$ is formed by obtaining the sensitivities of a desired output variable with respect to the states of the system. When the output variable of interest is the voltage magnitudes, $V_{i}$, with $i=1,2, \ldots, n$, with $n$ being the total number of buses of the network, the output matrix will be given by

$$
C_{V}=\left[\begin{array}{l|l}
C_{\mathrm{V} \delta} & C_{\mathrm{V} \omega}
\end{array}\right]
$$

where $C_{\mathrm{V} \delta}$ is given by the sensitivity of the voltage magnitude of the $i$-th bus of the power system with respect to the machine internal angles, i.e.

$$
\boldsymbol{C}_{\mathrm{V} \delta}=\left[\begin{array}{cccc}
\frac{\partial V_{1}}{\partial \delta_{1}} & \frac{\partial V_{1}}{\partial \delta_{2}} & \cdots & \frac{\partial V_{1}}{\partial \delta_{N}} \\
\vdots & \vdots & \ddots & \vdots \\
\frac{\partial V_{n}}{\partial \delta_{1}} & \frac{\partial V_{n}}{\partial \delta_{2}} & \cdots & \frac{\partial V_{n}}{\partial \delta_{N}}
\end{array}\right]
$$

A similar sensitivity matrix, $C_{\mathrm{V} \omega}$, can be obtained from the sensitivities of the voltage magnitudes with respect to the machine speeds.

Similarly, when the voltage angles $\theta_{i}$ are the output variables, the output matrix is given by

$$
\boldsymbol{C}_{\boldsymbol{\Theta}}=\left[\begin{array}{l|l}
\boldsymbol{C}_{\theta \delta} & \boldsymbol{C}_{\theta \omega}
\end{array}\right]
$$

Matrices $\boldsymbol{C}_{\theta \delta}$ and $\boldsymbol{C}_{\theta \omega}$ are obtained in similar fashion as done for obtaining (4).

Note that as discussed in Section II, the bus frequencies are a derived variable from the bus voltage angles. To obtain the bus frequency sensitivities we must apply a high-pass filter to each of the bus voltage angles [6]. For every individual filter the transfer function that defines it is given by

$$
H(s)=\frac{T s}{T s+1}
$$

where $s$ is the Laplace operator. The set of all the filters can be described by the state-space model

$$
\begin{aligned}
& \dot{\boldsymbol{x}}_{\mathrm{F}}=\boldsymbol{A}_{\mathrm{F}} \boldsymbol{x}_{\mathrm{F}}+\boldsymbol{B}_{\mathrm{F}} \boldsymbol{u}_{\mathrm{F}} \\
& \boldsymbol{y}_{\mathrm{F}}=\boldsymbol{C}_{\mathrm{F}} \boldsymbol{x}_{\mathrm{F}}+\boldsymbol{D}_{\mathrm{F}} \boldsymbol{u}_{\mathrm{F}}
\end{aligned}
$$

where $\boldsymbol{A}_{\mathrm{F}}, \boldsymbol{B}_{\mathrm{F}}, \boldsymbol{C}_{\mathrm{F}}$ and $\boldsymbol{D}_{\mathrm{F}}$ are the state space matrices of the diagonal set of filters and $\boldsymbol{x}_{\mathrm{F}}$ are the state vector, $\boldsymbol{u}_{\mathrm{F}}$ the control vector and $\boldsymbol{y}_{\mathrm{F}}$ the output vector of the filter system. 
Finally, the power system linearized model (1) is connected in series with the state space model of the filters (7) using the voltage angle outputs as inputs to the filter model, yielding the bus frequencies as the filter ouputs. The state space model of the series-connected system has the form

$$
\begin{aligned}
& \dot{\boldsymbol{x}}_{\mathrm{S}}=\boldsymbol{A}_{\mathrm{S}} \boldsymbol{x}_{\mathrm{S}}+\boldsymbol{B}_{\mathrm{S}} \boldsymbol{u}_{\mathrm{P}} \\
& \boldsymbol{y}_{\mathrm{S}}=\boldsymbol{C}_{\mathrm{S}} \boldsymbol{x}_{\mathrm{S}}+\boldsymbol{D}_{\mathrm{S}} \boldsymbol{u}_{\mathrm{P}}
\end{aligned}
$$

where the linearized matrices are given by

$$
\begin{gathered}
\boldsymbol{A}_{\mathrm{S}}=\left[\begin{array}{cc}
\boldsymbol{A}_{\mathrm{F}} & \boldsymbol{B}_{\mathrm{F}} \boldsymbol{C}_{\mathrm{P}} \\
\mathbf{0} & \boldsymbol{A}_{\mathrm{P}}
\end{array}\right], \boldsymbol{B}_{\mathrm{S}}=\left[\begin{array}{c}
\boldsymbol{B}_{\mathrm{F}} \boldsymbol{D}_{\mathrm{P}} \\
\boldsymbol{B}_{\mathrm{P}}
\end{array}\right] \\
\boldsymbol{C}_{\mathrm{S}}=\left[\begin{array}{ll}
\boldsymbol{C}_{\mathrm{F}} & \boldsymbol{D}_{\mathrm{F}} \boldsymbol{C}_{\mathrm{P}}
\end{array}\right], \boldsymbol{D}_{\mathrm{S}}=\left[\begin{array}{ll}
\boldsymbol{D}_{\mathrm{F}} & \boldsymbol{D}_{\mathrm{P}}
\end{array}\right]
\end{gathered}
$$

The output matrix from the series-connected system will include not only $C_{\mathrm{V} \delta}$ (4), but also the sensitivities of the bus frequencies with respect to the machine angles and speeds, i.e.

$$
\boldsymbol{C}_{\boldsymbol{f}}=\left[\begin{array}{l|l}
\boldsymbol{C}_{\mathrm{f} \delta} & \boldsymbol{C}_{\mathrm{f} \omega}
\end{array}\right]=\left[\begin{array}{ll}
\boldsymbol{C}_{\mathrm{F}} & \boldsymbol{D}_{\mathrm{F}} \boldsymbol{C}_{\Theta}
\end{array}\right]
$$

where $C_{\mathrm{F}}$ and $\boldsymbol{D}_{\mathrm{F}}$ are the output and feedforward matrices from the state space representation of the filters.

\section{B. Eigenvalues and Eigenvectors of the Linearized Model}

The eigenvalues and eigenvectors of matrix $\boldsymbol{A}_{\mathrm{S}}$ (8) yield two matrices: the diagonal matrix $\boldsymbol{\Lambda}$ containing the eigenvalues and the modeshape matrix $M$ containing the right-eigenvectors.

The right eigenvectors give the relative activity of the state variables when a mode is excited, i.e. the modeshapes, which are given by

$$
\boldsymbol{x}=\left[\begin{array}{lll}
M_{1} & \cdots & M_{m}
\end{array}\right] \boldsymbol{z}
$$

where $\boldsymbol{M}_{i}$ is the $i=1,2, \ldots, m$ eigenvector of the system, and $z$ is a transformed state variable such that each variable is associated with only one mode.

\section{Bus Voltage Magnitude and Frequency Oscillations}

We now establish an important relationship between the linearized model (8) and the oscillations described in Section II. We can multiply each voltage output matrix with the right eigenvectors to give a measure of the variations in bus voltage and frequency when a particular mode is excited, primarily interarea modes. For the bus frequency variations the bus frequencies are derived from the bus voltage angles.

We can define then the bus voltage magnitude oscillations as the variation of the voltage magnitude at each system bus with respect to an excited mode. Similarly, the bus frequency oscillations can be defined as the variation of the bus frequency with respect to the excited mode. The bus voltage mangitude variation gives a measure of the amplitude of the oscillations at any given bus. With this measure it is possible, among other several applications, to locate good signals for monitoring and control design, and to determine the points of maximum and minimum oscillation of the voltage that can be used to extrapolate to the network's fixed point ([6]).
The bus voltage magnitude oscillations are computed by using the eigenvector matrix $M$ as follows

$$
\Delta V=C_{\mathrm{V} \delta} M
$$

In addition the frequency oscillations will provide a measure of th variation of the bus frequency with respect to the machine angles, they are computed with

$$
\Delta \boldsymbol{F}=\boldsymbol{C}_{\mathrm{f} \delta} M
$$

The oscillations computed by (12) and (13) contain all modal components, including the interarea modes. For this investigation we focus on the voltage and frequency oscillations from the interarea modes only. In the sequel we apply the analytical method described above to multimachine systems.

\section{Application to a Two-Machine Radial System}

Here we apply the method outlined in Section III to a twomachine radial system as depicted in Fig. 1, and also used in [6], [8]. The system is subject to different loading cases, $\mathrm{C} 1: 7 \mathrm{pu}, \mathrm{C} 2: 5 \mathrm{pu}$, and $\mathrm{C} 3: 0.35 \mathrm{pu}$ of power flow from $G_{1}$ to $G_{2}$. Figure 4 a shows the voltage oscillations and Fig. $4 \mathrm{~b}$ the frequency oscillations computed for the two machine systems. Note that there are more data points in the plots than power system buses define in the one-line diagram, this is because the line reactances have been segmented to obtain a better resolution of the oscillation curves (i.e. there are more intermediate buses between $G_{1}$ and $G_{2}$ in the model used). Because the method computes the oscillations at the network

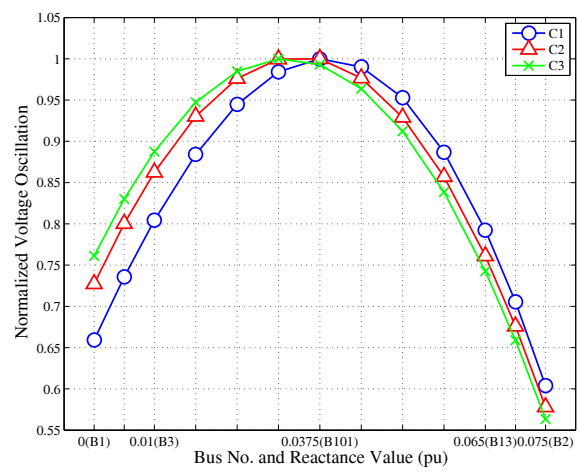

(a) Normalized Bus Voltage Magnitude Oscillations

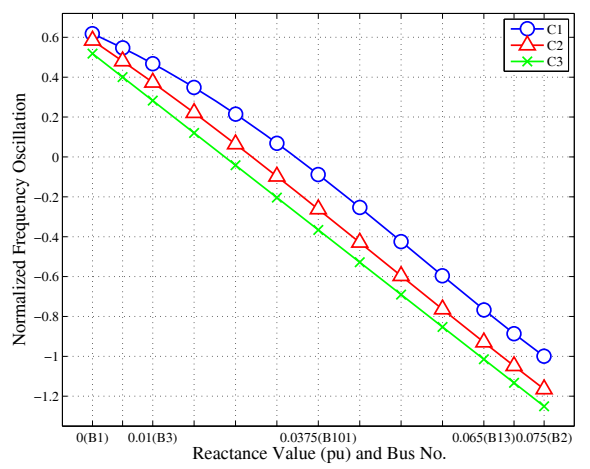

(b) Normalized Bus Frequency Oscillations

Figure 4. Normalized Voltage Magnitude and Bus Frequency Oscillations in the Two-Machine Radial System. Power Transfer Levels: C1: 7 pu, C2: 5 $\mathrm{pu}$, and C3: $0.35 \mathrm{pu}$. 
buses, the curves do not explicitly extend to the internal nodes of the machines. They can easily be extended using a curve fitting method.

Observe that, as the loading of the system is decreased the peak of the normalized voltage oscillations moves to the left. The intersection point of the frequency oscillations curve with the $x$-plane also moves to the left. This can be viewed as the internal machine voltage fixed point adjusting to the line loading. For Case $\mathrm{C} 1$ the reactance of $G 1$ will be much larger than that of $G_{2}$ per the adjustment of the voltage fixed point. The frequency oscillation propagation is also affected by the loading, even though the intersection of the curves with the $x$-plane is shifted if the curves are extended into the internal machine nodes the ratios between $G_{1}$ and $G_{2}$ will be maintained. This can be viewed as the center of the network moving to balance the power loading and the inertias.

\section{Application to a Four-Machine System}

In this section we compute the voltage and frequency oscillations from the interarea mode of the familiar two-area system ([2], [5]) shown in Fig. 5, and also used in [6], [8]. Here the machines have been modeled with the electromechanical model, and the transmission lines have been segmented to provide for more resolution in the oscillation curves. The network possesses one interarea mode between Area $1\left(G_{11}\right.$ and $\left.G_{12}\right)$ and Area $2\left(G_{21}\right.$ and $\left.G_{22}\right)$, a local mode within Area 1 and a second local mode within Area 2.

The system is subject to different load flow conditions that change the power flow of the tie lines between Buses 3-101-13. This is attained by changing the loads $L_{4}$ and $L_{14}$ accordingly as shown in Table I.

Figures $6 \mathrm{a}$ and $6 \mathrm{~b}$ show the normalized voltage and frequency oscillations across the transmission path formed by Buses 20-3-101-13-120. The oscillation curves are computed for the different load cases shown in Table I, in Case C1 the transfer path is highly loaded whereas in Case C4 the transmission path has no power transfer. Observe from Fig.

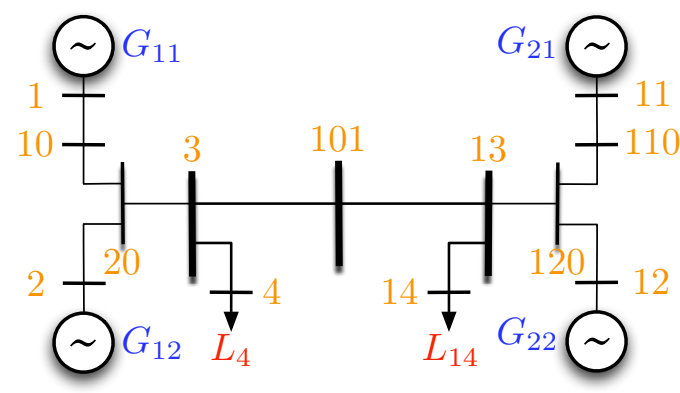

Figure 5. Four-Machine Power System

Table I

LOAD CONFIGURATIONS FOR THE FouR-MACHINE SySTEM

\begin{tabular}{|c|c|c|}
\hline Case No. & $L_{4}(\mathrm{pu})$ & $L_{14}(\mathrm{pu})$ \\
\hline \hline C1 & 10.7 & 16.7 \\
C2 & 11.7 & 15.7 \\
C3 & 12.7 & 14.7 \\
C4 & 13.7 & 13.7 \\
\hline
\end{tabular}

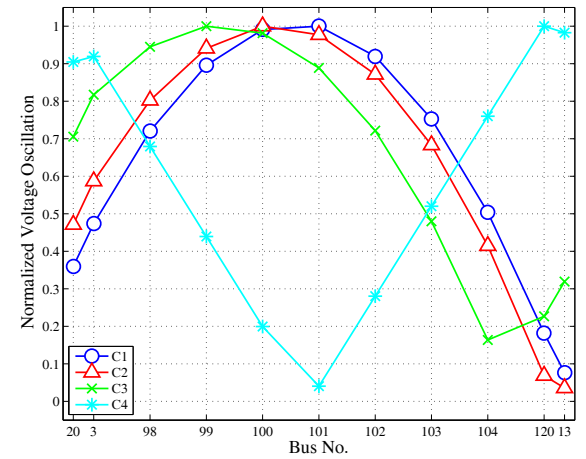

(a) Normalized Bus Voltage Magnitude Oscillations

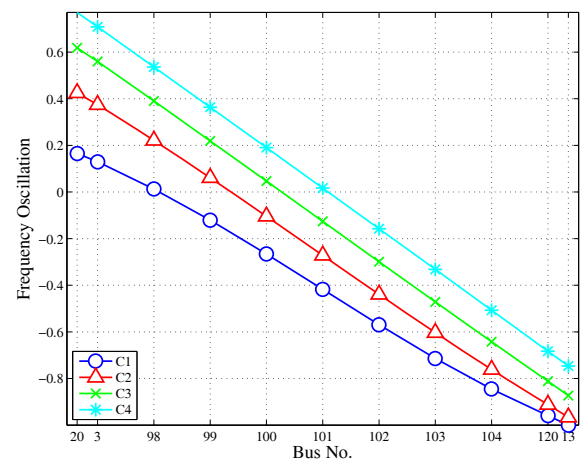

(b) Normalized Bus Frequency Oscillations

Figure 6. Comparison of Bus Voltage Magnitude and Bus Frequency Oscillations from Interarea Modes in the Transmission Path 20-3-101-13-120

$6 \mathrm{a}$ that, as the loading of the transfer path is decreased, the peak of the normalized voltage oscillation curves moves to the left. Similarly to the two-machine system, we can conceptualized two aggregated generators in each area, and interpret the resulting machine voltage fixed point adjusting for the different power levels. The analogy with the two-machine system cannot be extended completely for the frequency oscillations as the inertias of the four-machine system are different. Nevertheless, the frequency oscillations also exhibit a shift in the intersection with the $x$-plane. We view this as a fact that the midpoint will shift to balance for the loading within each power transfer path.

In Figures 7 and 8, a projection of the computed voltage magnitude and frequency modeshapes for the interarea mode are shown for Cases 1 and 3. The yellow circles are used to denote major transmission buses, which are connected by a solid black line representing the transmission lines. The computed oscillations at each system bus are represented by the dotted blue lines accompanied by black-filled circles, while magenta stems show the oscillations for load buses.

For the interarea mode the voltage oscillations are the highest at the transfer path. Observe that in the case of the local modes this will not be the case. The voltage modeshapes for branches 2-20-10-1 and 12-120-110-11 have a very similar behavior across each branch, their maximum values observed at Buses 20 and 120 are prescribed by the oscillations coming from the dominant path. Finally, for the frequency oscillations, the projection plot provides a better visualization of how the frequency oscillations propagate. Observe that they are mostly 
Case $\mathrm{C} 1: \mathrm{L}_{4}=10.7 \& \mathrm{~L}_{14}=16.7$

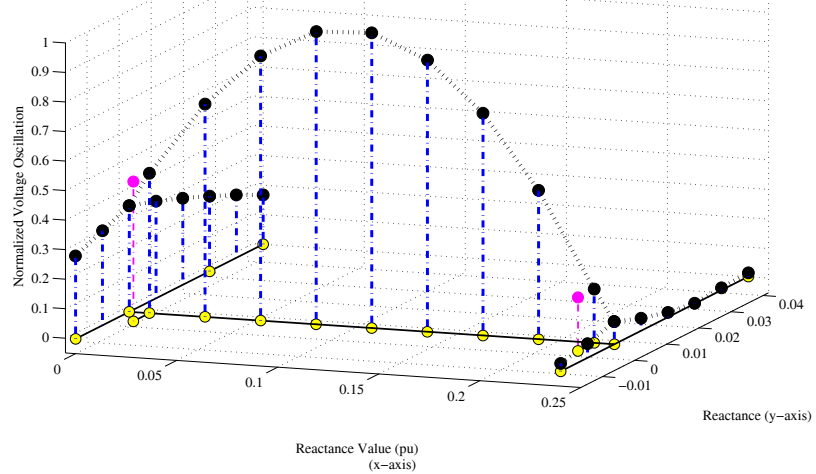

(a) Projection of the Bus Voltage Magnitude Oscillations for Case C1

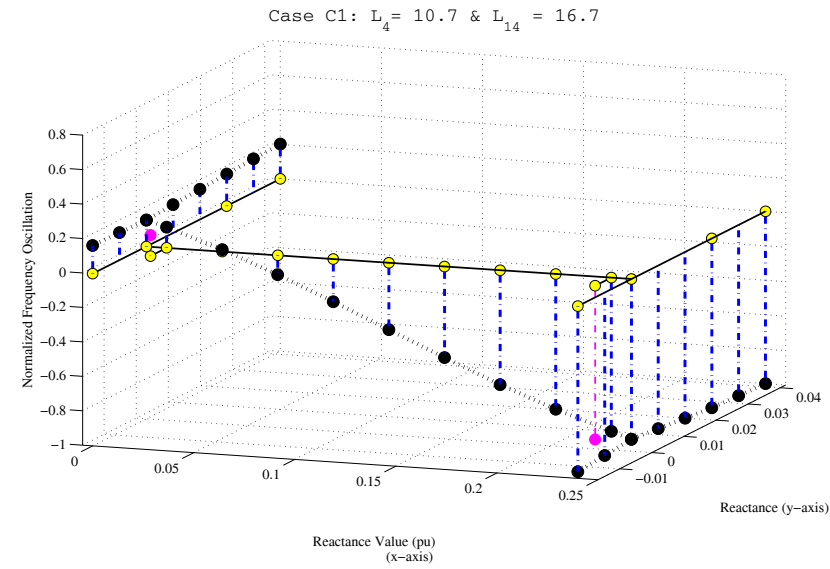

(b) Projection of the Bus Frequency Oscillations for Case C1

Figure 7. Projection of the Bus Voltage Magnitude and Bus Frequency Oscillations from the Interarea Mode in the Two-Area System - Case C1

constant at each area with minimal variation. Future analysis will provide insight on how to understand these effects from multiple machine inertias.

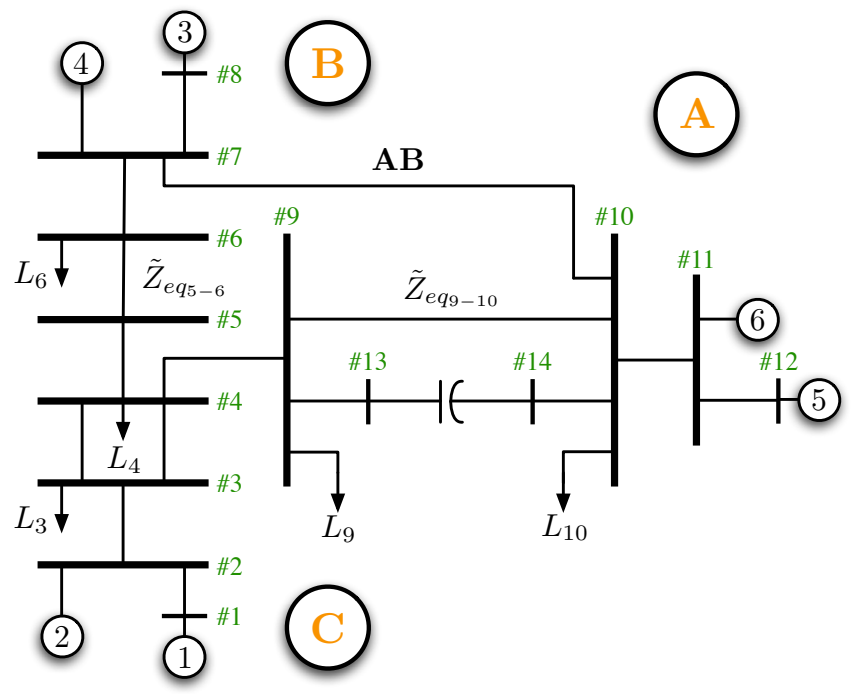

Figure 9. Six-Machine Power System
Case $\mathrm{C} 3: \mathrm{L}_{4}=12.7 \& \mathrm{~L}_{14}=14.7$

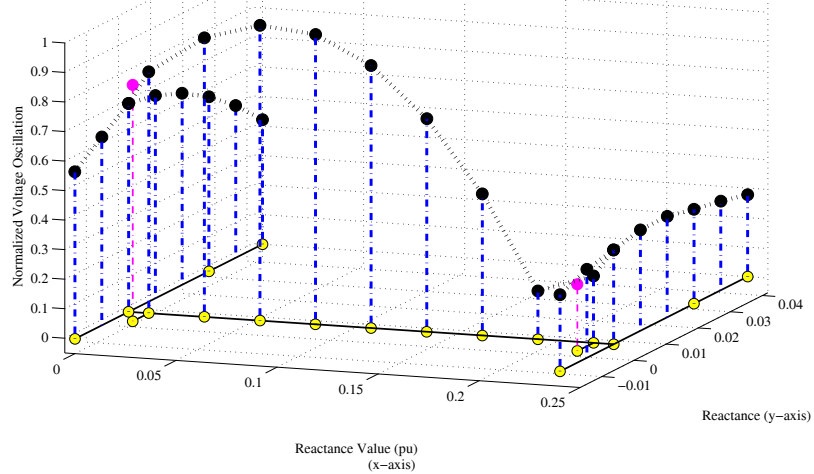

(a) Projection of the Bus Voltage Magnitude Oscillations for Case C3

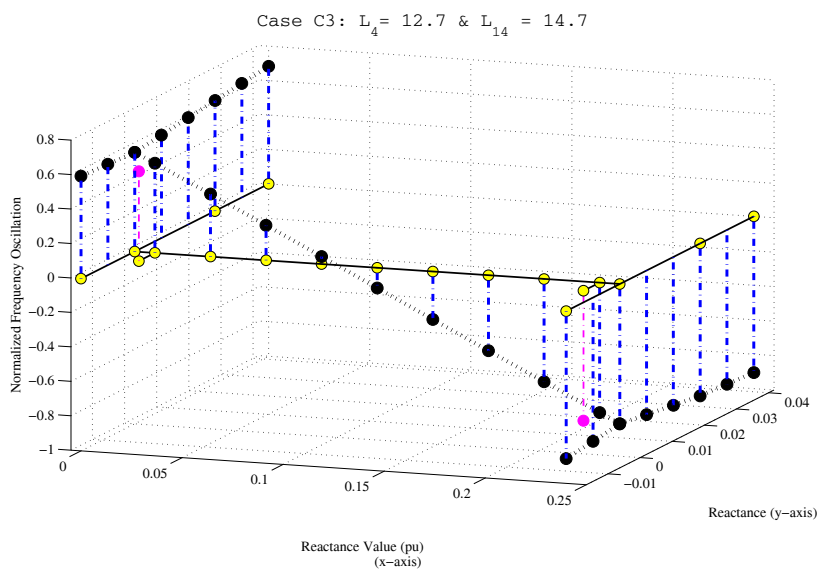

(b) Projection of the Bus Frequency Oscillations for Case C3

Figure 8. Projection of the Bus Voltage Magnitude and Bus Frequency Oscillations from the Interarea Mode in the Two-Area System - Case C3

\section{Vi. Application to a Six Machine System}

This Section presents preliminary results of the application of the analytical method to the six-machine power system (modified from [9]) shown in Fig. 9. This system has multiple interarea modes: one between areas A and B that is dominant through the lines 7-10 and 9-10, and a second mode that has the most impact between areas $\mathrm{B}$ and $\mathrm{C}$ through buses 1 to 8. Figure 10 and 11 show the oscillations from the interarea mode $\mathrm{B}$ to $\mathrm{C}(\mathrm{BC})$ and $\mathrm{A}$ to $\mathrm{B}(\mathrm{AB})$, respectively.

\section{A. BC Interarea Mode}

The voltage oscillations of interarea mode BC (Fig. 10a) are the maximum between the buses from 1 to 8 where the mode propagates the most, the general shape of the curve deviates from the shape in the two-machine system because is subject to the effect of intermediate loads. Observe also that the path between nearby machines (i.e. generators $1 \& 2$ and $3 \& 4$ ) shows the distinctive Jacobian shape as this part of the network is not affected by intermediate loads. Another important observation is to realize that the within the path that an interarea mode propagates several of these Jacobian type curves may emerge. It is important to note that the mode also propagates, although with less intensity in the line 7-10 where 
the Jacobian pattern repeats. The effects of the propagation of the oscillations are more difficult to interpret in path 9-10 due to the presence of parallel elements (transmission lines and series capacitor). The influence of this elements is more apparent in the AB interarea mode discussed below.

The frequency oscillations (Fig. 10b) show the same prominent characteristic observed in the other two systems analyzed. The most important observation to make is that the frequency crossover will take place in the dominant oscillation path, in this case from Bus 1 to 8 . The frequency oscillation curve from the dominant transfer path will set the departing frequencies in the other paths which will follow the trend of that oscillation curve (increase of decay of oscillation).

\section{$B$. AB Interarea Mode}

For this mode, the behavior of the voltage oscillations (Fig. 11a) is more difficult to interpret, as it is influenced by different transfer paths. The mode is distributed between three different paths: AB, $\tilde{Z}_{e q_{9-10}}$, and the series-compensated line. The mode has the highest oscillations at path $\mathrm{AB}$, with the same behavior seen in the other two power systems analyzed.

The voltage oscillations in the $\tilde{Z}_{e q_{9-10}}$ path exhibit a high influence of the loads $L_{9}$ and $L_{10}$, as seen in the BC mode this effect makes the shape different from the Jacobian pattern. The voltage oscillations across the series-compensated line has been indicated by a combination of cyan, magenta, and green filled dots, the magenta dots correspond to the series capacitor. A profile view is shown in Fig. 12. Observe that the effect of the loads are less prominent and that the oscillations propagate with a pattern similar to the Jacobian fit.

It can be noted from Fig. 11b that the frequency oscillations behave more regularly as seen in interarea mode $\mathrm{BC}$ and the other two power systems. Observe also that differently from the voltage oscillations, the frequency oscillations are better distributed between the three paths.

\section{CONCLUSIONS}

This paper has focused on the development of an analytical method to compute voltage and frequency oscillations starting from a linearized power system model. Applications to a twomachine, and a four-machine system with single interarea

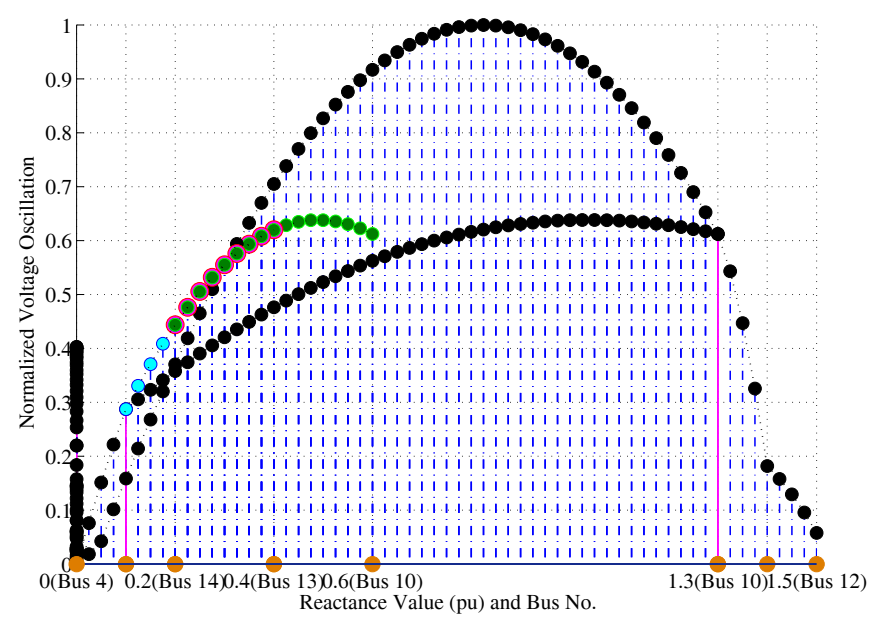

Figure 12. Profile view of the Interarea Mode No. 2 voltage oscillations mode has been presented. Application to a six-machine system with multiple interarea modes provides a new way of analyzing how voltage and frequency oscillations propagate in systems with more than one interarea mode. The visualization of these modes using projections into three-dimensional plots allows for better understanding of the propagation of these oscillations. This work should be seen as a starting point on how to understand and analyze these type of network variables within modal analysis. Future research will focus on further understanding of the underlying oscillation characteristics, and the effect of voltage control devices such as SVC and TCSC.

\section{ACKNOWLEDGMENT}

This work is supported in part by the RPI Power System Research Consortium Industry Members: AEP, FirstEnergy, ISO NE, NYISO, and PJM, and by NSF through grant ECS0622119.

\section{REFERENCES}

[1] J. H. Chow, G. Peponides, P. V. Kokotovic, B. Avramovic, and J. R. Winkelman, Time-Scale Modeling of Dynamic Networks with Applications to Power System, J. H. Chow, Ed. New York: Springer-Verlag, 1982.

[2] M. Klein, G. J. Rogers, and P. Kundur, "A Fundamental Study of Inter-Area Oscillations in Power Systems," IEEE Transactions on Power Systems, vol. 6, no. 3, pp. 914-921, Aug. 1991.

[3] G. Verghese, I. Perez-Arriga, and F. Schweppe, "Selective Modal Analysis with Application to Electric Power Systems, Part I: Heuristic Introduction, Part II: The Dynamic Stability Problem," IEEE Transactions on Power Apparatus and Systems, vol. PAS-101, pp. 3117-3134, 1982.

[4] P. Kundur, Power System Stability and Control, ser. The EPRI Power System Engineering Series, N. J. Balu and M. G. Lauby, Eds. McGrawHill, 1994.

[5] G. Rogers, Power System Oscillations. MA: Kluwer, December 1999.

[6] J. Chow, A. Chakrabortty, L. Vanfretti, and M. Arcak, "Estimation of radial power system transfer path dynamic parameters using synchronized phasor data,' IEEE Transactions on Power Systems, vol. 23, no. 2, pp. 564-571, May 2008.

[7] J. H. Chow, L. Vanfretti, A. Armenia, S. Ghiocel, S. Sarawgi, N. Bhatt, D. Bertagnolli, M. Shukla, X. Luo, D. Ellis, D. Fan, M. Patel, A. M. Hunter, D. E. Barber, and G. L. Kobet, "Preliminary synchronized phasor data analysis of disturbance events in the us eastern interconnection," IEEE PES Power Systems Conference and Exposition, 2009. PSCE '09.' pp. 1-8, March 15-18 20092009.

[8] L. Vanfretti, "Notions of phasor measurement-based power system model reduction lf large power systems," M.Sc. Thesis, Rensselaer Polytechnic Institute, Troy, New York, July 2007.

[9] J. Shiau, G. Taranto, J. Chow, and G. Boukarim, "Power swing damping controller design using an iterative linear matrix inequality algorithm," IEEE Transactions on Control Systems Technology, vol. 7, no. 3, pp. 371-381, May 1999.

Luigi Vanfretti Luigi Vanfretti (S'03) received the Electrical Engineering degree from Universidad de San Carlos de Guatemala in 2005. He is pursuing the Ph.D. degree in the Electrical, Computer, and Systems Engineering Department at Rensselaer Polytechnic Institute, from where he obtained his MS in 2007. He was a visiting researcher at the Department of Electronics and Electrical Engineering of The University of Glasgow, Scotland, in Fall 2005. His research interests are modeling, dynamics, and stability of power systems; applications of PMU data and open source software for power system analysis.

Joe H. Chow (F'92) received his MS and $\mathrm{PhD}$ degrees from the University of Illinois, Urbana-Champaign. After working in the General Electric Power System business in Schenectady, he joined Rensselaer Polytechnic Institute in 1987. He is a professor of Electrical, Computer, and Systems Engineering and the Associate Dean of Engineering for Research and Graduate Programs. His research interests include multivariable control, power system dynamics and control, voltage-sourced converter-based FACTS Controllers, and synchronized phasor data. 


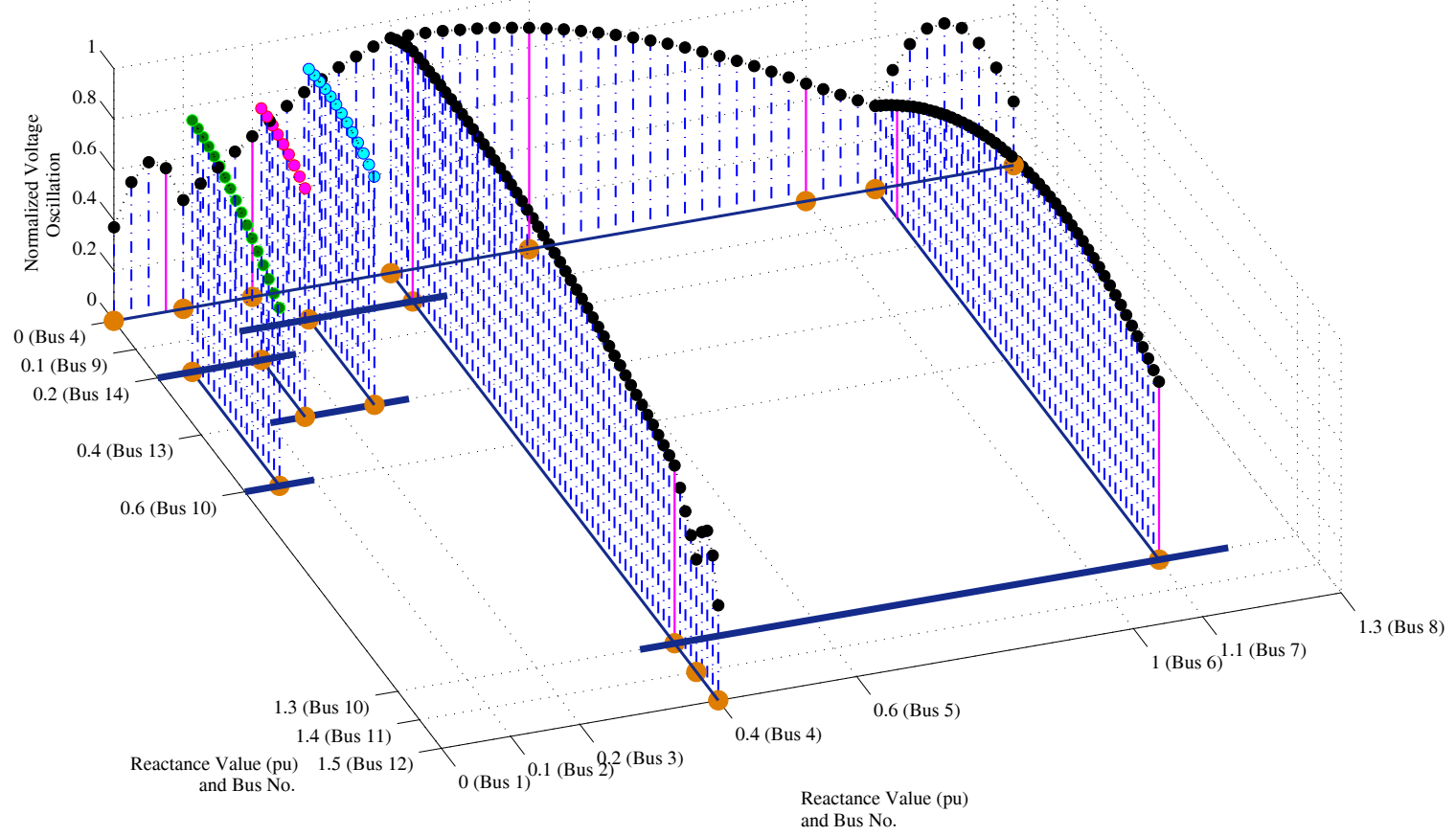

(a) Projection of the Bus Voltage Magnitude Oscillations

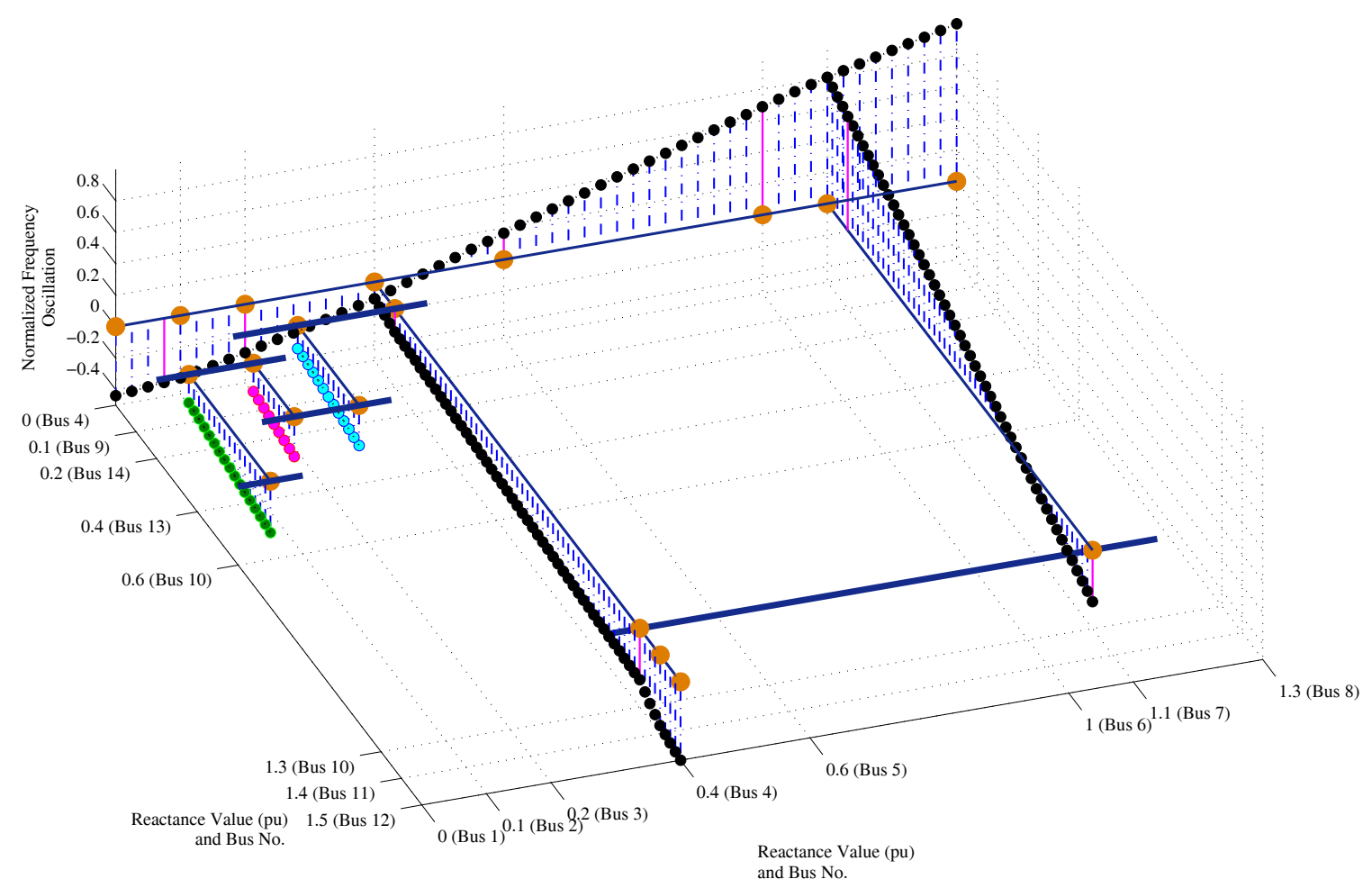

(b) Projection of the Bus Frequency Oscillations

Figure 10. Projection of the Bus Voltage Magnitude and Bus Frequency Oscillations from Interarea Mode No.1 in the Six-Machine System 


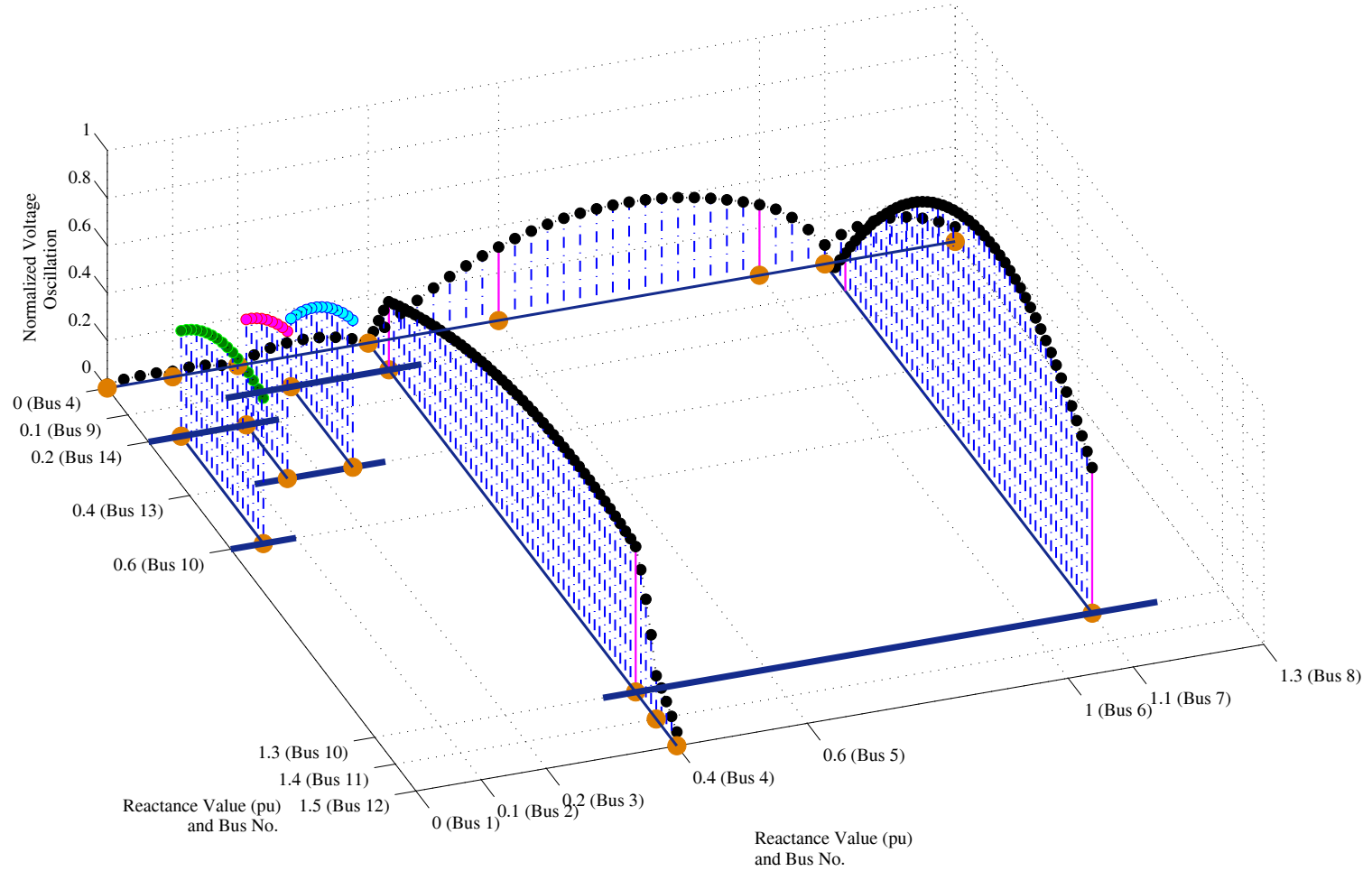

(a) Projection of the Bus Voltage Magnitude Oscillations

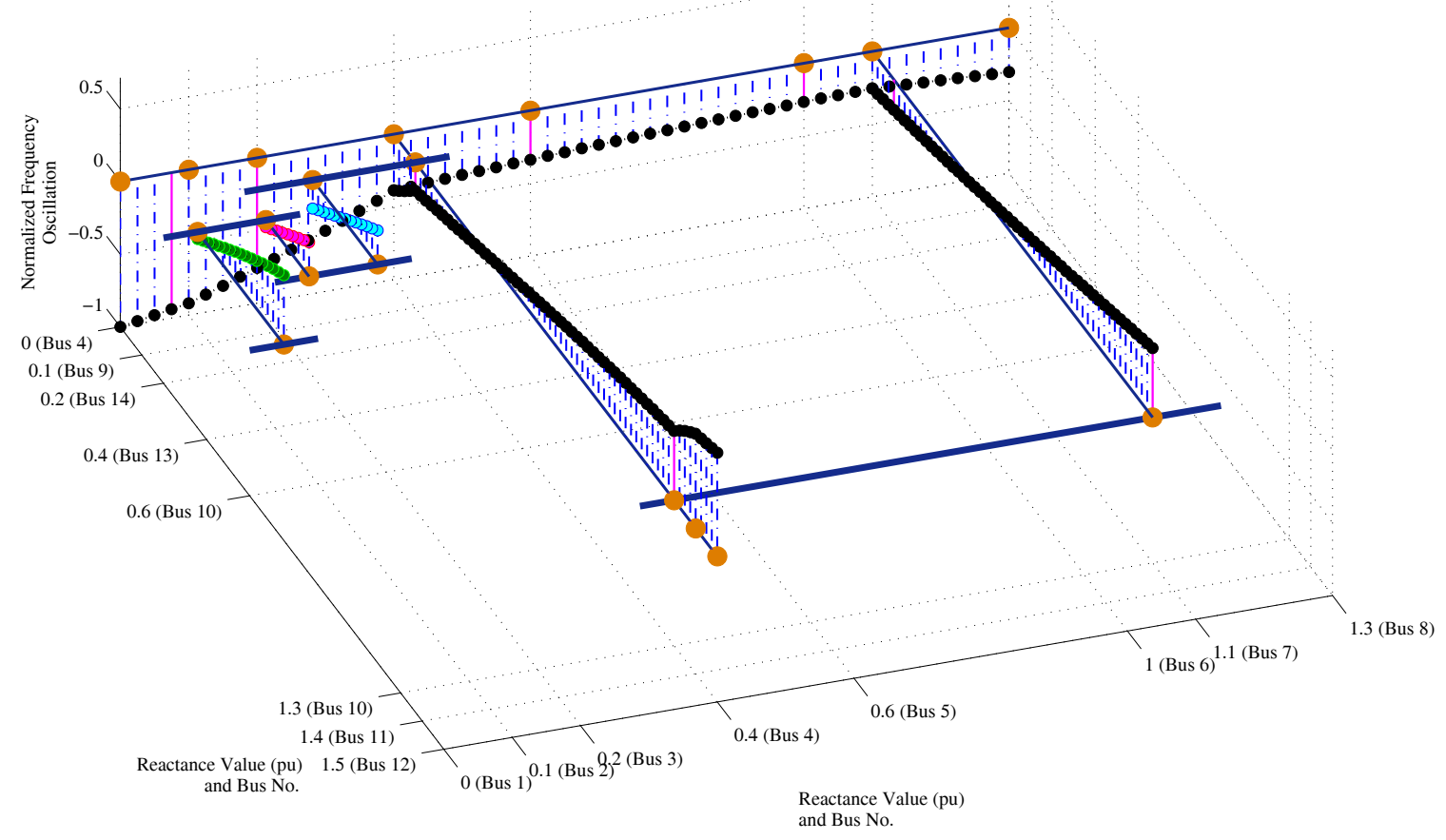

(b) Projection of the Bus Frequency Oscillations

Figure 11. Projection of the Bus Voltage Magnitude and Bus Frequency Oscillations from Interarea Mode No. 2 in the Six-Machine System 